\title{
Optical manipulation of ultrafast electron and nuclear motion on metal surfaces
}

\author{
Hrvoje Petek (petek@pitt.edu) \\ Department of Physics and Astronomy \\ University of Pittsburgh \\ Pittsburgh, PA 15269
}

We study the unoccupied electronic structure and dynamics of chemisorbed atoms and molecules on metal surfaces by time resolved two-photon photoemission (TR-2PP) spectroscopy, low temperature scanning tunneling microscopy (LT-STM), and theory. ${ }^{1-9}$ Our research concerns simple atomic adsorbates such as alkali and alkaline earth atoms, which provide fundamentally important models for adsorbate-surface interactions, and more complex adsorbates such as fullerenes on noble metals, which illustrate emergent interfacial properties that derive from intrinsic molecular attributes, and moleculemolecule and molecule-surface interactions. Our goal is to understand how these interactions contribute to formation of the interfacial electronic structure, and how thus formed electronic properties affect interfacial phenomena of importance to energy transduction and storage. Moreover, we explore how the interfacial electronic excitation drives dynamical phenomena such as charge transfer and surface femtochemistry.

Electronic structure of a chemisorption interface. We performed systematic studies of the electronic structure of alkali ( $\mathrm{Li}, \mathrm{Na}, \mathrm{K}, \mathrm{Rb}$, and $\mathrm{Cs}$ ) and alkaline earth (Ba) atoms chemisorbed on $\mathrm{Cu}(111)$ and $\mathrm{Ag}(111)$ surfaces. Surface normal and angleresolved two-photon photoemission (2PP) spectra were measured with $10 \mathrm{fs}, 3.1 \mathrm{eV}$ photon energy laser pulses. At $<0.1$ monolayer coverage used our experiment, alkali atoms chemisorb in an ionic state. Surprisingly, the unoccupied valence $n s$ resonance of alkali atoms was found to be at 3.0 and $2.8 \mathrm{eV}$ above the Fermi level $\left(E_{F}\right)$ on $\mathrm{Cu}(111)$ and $\mathrm{Ag}(111)$ surfaces, respectively. This universal behavior was independent of the atomic size and ionization potential within the alkali atom group. We could explain this unexpected behavior with a simple model, which considers the dominant polarization interaction between alkali atoms and metal surfaces. The model we developed should be applicable to predicting the occupied and unoccupied electronic structures of adsorbates on metal surfaces, unless the nature of the interaction has a strong covalent character.

In our model, an alkali atom and a metal surface interact through the Coulomb potential. The conduction electrons in the metal substrate screen the charges of $n s$ valence electron and alkali atom core thereby creating the oppositely charged image charges in the substrate. Thus, the energy of $n s$ valence electron near a metal surface is defined by the Coulomb attraction to the positive core and its image charge, and the repulsion by the image charge of the core. The net repulsive potential destabilizes the $n s$ electron with $1 / 4 z$ dependence, where $z$ is the surface normal distance from the image plane, as alkali atom is brought from vacuum to its chemisorption height $R_{a d s}$ above the metal surface. Lifting the $n s$ electron above $E_{F}$ enables its tunneling into the conduction band of the substrate. The bound state state-continuum interaction between the localized atomic orbitals and the propagating states of the substrate renders the $n s$ level into a $\sigma$-resonance ( $m=0$, where $m$ is the surface projection of the angular momentum quantum number $l$ ) in the unoccupied electronic structure of the interface. The $\sigma$-resonance appears as a prominent feature in 2PP spectra of alkali atom covered noble metal surfaces. ${ }^{7}$ 
We have calculated the $\sigma$ resonance energies of alkali atoms on $\mathrm{Cu}(111)$ and $\mathrm{Ag}(111)$ surfaces using a wave packet propagation procedure and a simple model that simply ascribes them to the $1 / 4 z$ repulsion. Both procedures reproduce the period independent $\sigma$ resonance energy, implying that there is an anticorrelation relationship between the atomic ionization potential and $R_{a d s}$. The binding energy of $n s$ electron and $R_{a d s}$ are determined by how strongly the core electrons of an alkali atom screen the Coulomb potential of its nucleus. Small atoms with ineffectively screened nucleus (e.g. Li) strongly bind the $n s$ electron and can approach close to the surface to a

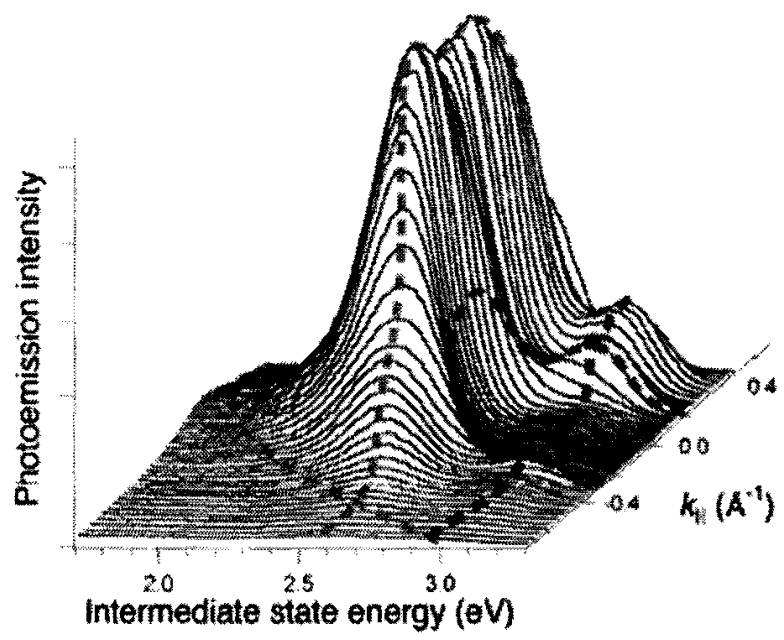

Figure 1. Angle resolved 2PP spectra of $\mathrm{Cs} / \mathrm{Cu}(111)$. The blue and black dashed lines indicate the $\sigma$ - and $\pi$-resonances. ${ }^{8}$ distance defined by the Pauli exclusion. The degree of $n s$ electron repulsion, however, also depends on $R_{a d s}$ counteracting the strong binding. The correspondingly weak interactions for large alkali atoms result in the period independent $\sigma$-resonance energy.

$\pi$-resonances. Following the successful explanation of the $\sigma$-resonance energy, we reasoned that higher resonances should also be observable for alkali atoms on noble metal surfaces. Therefore, we investigated the angle dependent $2 \mathrm{PP}$ spectra in search of $\mathrm{m}=1$ resonances arising from the hybridization of the $n p_{x}$ and $n p_{y}$ atomic orbitals. Indeed we found a new resonance at $0.4-0.7 \mathrm{eV}$ above the $\sigma$-resonances, when observing emission in the off-normal direction. Figure 1 shows a series of typical angle resolved $2 \mathrm{PP}$ spectra of $\mathrm{Cs} / \mathrm{Cu}(111)$ surface, where the main features are the $\sigma$ - and $\pi$-resonances of Cs. The $\sigma$-resonance has maximum intensity near the normal emission, while the $\pi$ resonance peaks at $\pm 15-20^{\circ}$ from the normal. This difference is explained by their respective $m=0$ and $m=1$ symmetries; the $m=1$ states have zero emission in the normal direction because of nodes in their wave functions. Interestingly, we did not observe $\pi$ resonances for $\mathrm{Na}$. Our interpretation is that such resonances exist at higher energies for $\mathrm{Li}$ and $\mathrm{Na}$, because for $\mathrm{K}$ and larger alkali atoms the hybridization between the $n p$ and $n d$ atomic orbitals helps to stabilize the $\pi$-resonances. ${ }^{8}$

Ba chemisorption. We also investigated the electronic structure of $\mathrm{Ba} / \mathrm{Cu}(111)$ surface in order to ascertain the differences between chemisorption of one and two valance electron atoms. Indeed, we observe a prominent $\sigma$-resonance at $2.2 \mathrm{eV}$ above $E_{F}$ in the zero coverage limit. Besides the lower resonance energy, the bandwidth of $0.5 \mathrm{eV}$ is considerably broader than, for example, the $\sigma$-resonance of its neighboring element $C s$.

In order to gain an understanding of how the alkali and alkaline earth chemisorption differs, we performed electronic structure calculations of both $\mathrm{Cs}$ and $\mathrm{Ba}$ using an embedding approach with a one-dimensional potential for the surface, which quantitatively reproduces the experiment. The differences between the electronic structures of $\mathrm{Cs}$ and $\mathrm{Ba}$ on the $\mathrm{Cu}(111)$ surface can be attributed to the group dependent screening of the core potentials as manifested by the ionic radii and ionization potentials (alkali vs. alkaline earth). We find that in both cases one $6 s$ electron is transferred to the 
Figure 2. Spatial distribution of the electronic charge density for $\mathrm{Cs} / \mathrm{Cu}(111)$ (upper panel) and $\mathrm{Ba} / \mathrm{Cu}(111)$ (lower panel). The origin (cross) corresponds to the position of the adatom. Vertical lines indicate the image plane position. ${ }^{9}$

substrate, which, in the case of $\mathrm{Ba}$, leaves the second electron still localized on the substrate. The partial charge transfer of $\mathrm{Ba}$ valence electrons is responsible for the differences between the calculated electronic charge densities of $\mathrm{Cs}$ and $\mathrm{Ba}$ on $\mathrm{Cu}(111)$, which are shown in Fig. 2. Interestingly, the simple model used to calculate the energy $\sigma$-resonances of alkali atoms works equally well for $\mathrm{Ba}$ under the assumption that a single electron is transferred to the substrate. ${ }^{9}$

Superatom states of hollow molecules. We used LT-STM and density functional theory to explore

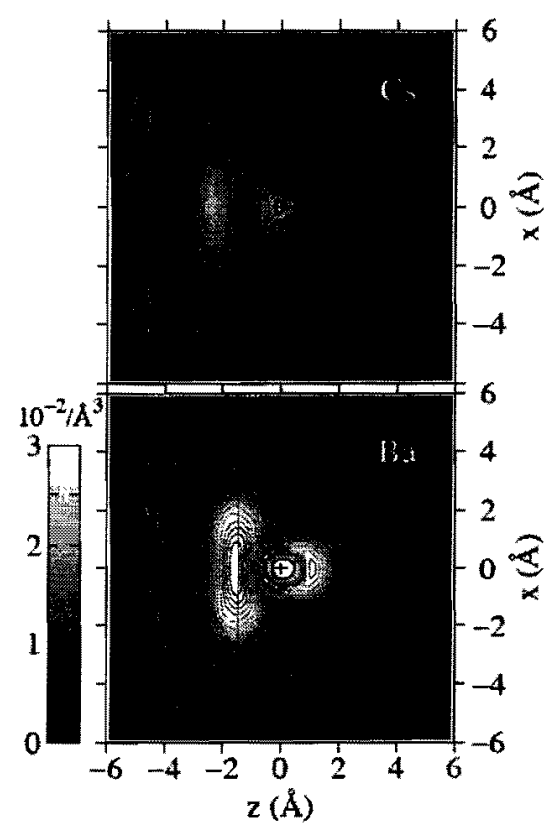
the relation between the nearly spherical shape and unoccupied electronic structure of $\mathrm{C}_{60}$ molecules adsorbed on copper surfaces. Above the known $\pi^{*}$ antibonding molecular orbitals of the carbon-atom framework, starting from $3.5 \mathrm{eV}$ we found atomlike orbitals bound to the core of the hollow $\mathrm{C}_{60}$ cage (Fig. 3 ). These "superatom" states hybridize like the $s$ and $p$ orbitals of alkali atoms into diatomic molecule-like dimers and free-electron bands of one-dimensional wires and two-dimensional quantum wells in $\mathrm{C}_{60}$ aggregates. We attribute the superatom states to the central potential binding an electron to its screening charge, a property expected for hollow-shell molecules derived from layered materials. The atomic electron orbitals that underlie molecular bonding originate from the central Coulomb potential of the atomic core. Because the superatom states originate from the image potential of graphene, they are a general feature derived from rolling and wrapping of molecular sheets into hollow molecules. ${ }^{5}$

Future plans. The interaction of alkali atoms with graphitic materials and metal oxides is important in energy storage applications such as rechargeable $\mathrm{Li}$ ion batteries. We have therefore initiated a study of the alkali atom electronic structure on HOPG and
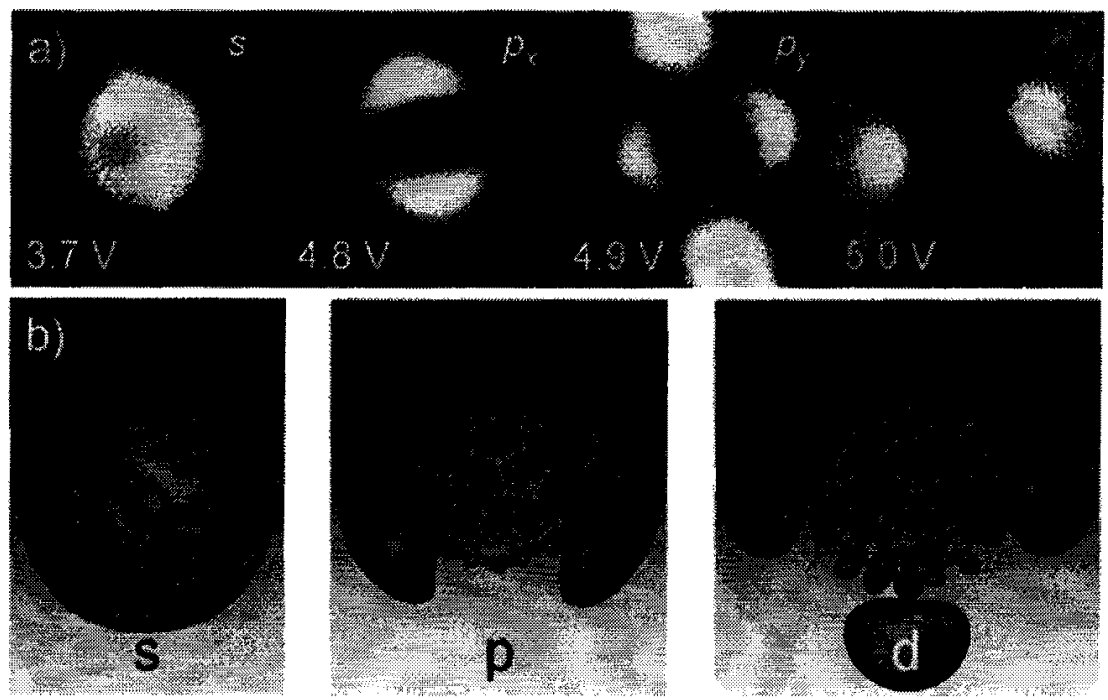

Figure 3. a) The observed $s, p$, and $d$ superatom orbitals of $\mathrm{C}_{60}$ on $\mathrm{Cu}(110)-\mathrm{O}$ surface. b) the corresponding calculated orbitals from a plane-wave DFT calculation for an isolated $\mathrm{C}_{60}$

molecule. $^{5}$
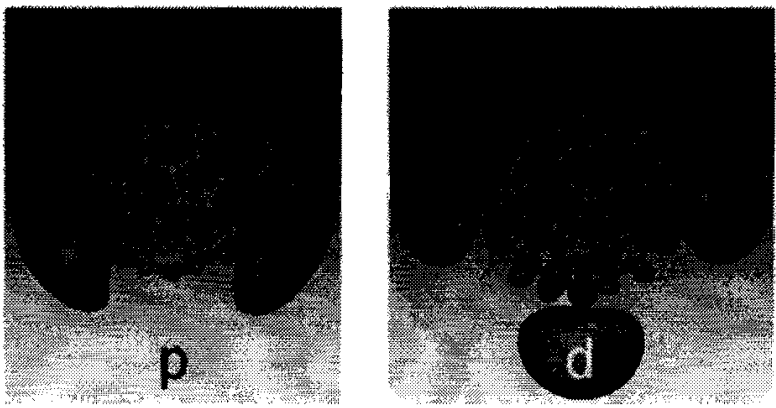
$\mathrm{TIO}_{2}$. Our preliminary studies for HOPG substrates show evidence for $n s$ states of alkali atoms. The samples show complex temperature and alkali atom period dependent behavior that could be related to intercalation or photodesorption. Moreover, we are investigating the effect of endohedral doping of fullerenes on the energy of superatom states. In preliminary measurements on $\mathrm{Sc}_{3} \mathrm{~N} @ \mathrm{C}_{80}$ we have found evidence for the superatom states at higher energy than for $\mathrm{C}_{60}$ (Fig. 4), which is consistent with the endohedral cluster excluding the superatom states from the internal volume. ${ }^{10}$
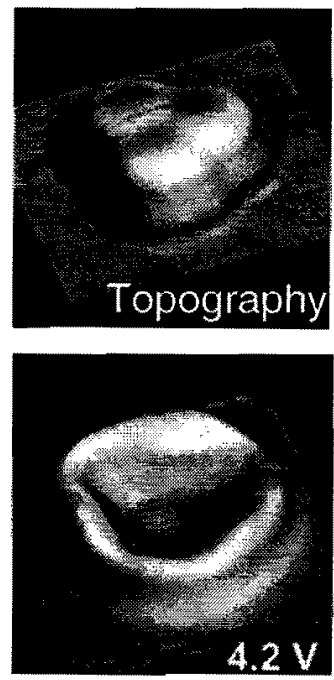
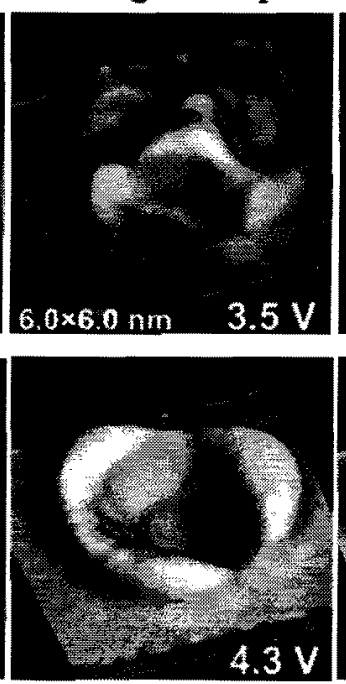
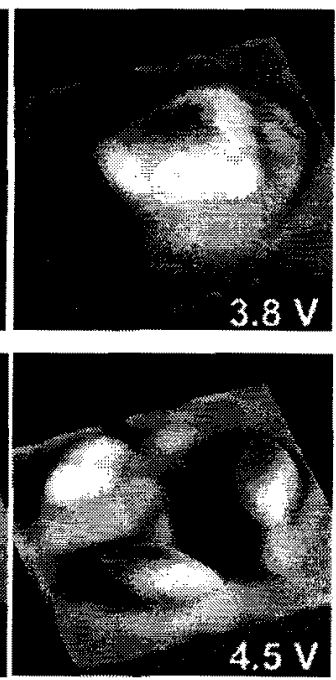

Figure 4. The topography of an equilateral $\mathrm{Sc}_{3} \mathrm{~N} @ \mathrm{C}_{80}$ trimer and $\mathrm{dI} / \mathrm{dV}$ maps at several representative voltages. The superatom orbitals hybridize into molecular orbitals of the aggregate that resemble those of an alkali atom trimer.

1. M. Fuyuki, K. Watanabe, D. Ino, H. Petek, and Y. Matsumoto, "Electron-phonon coupling at an atomically defined interface: Na quantum well on $\mathrm{Cu}(111)$," Phys. Rev. $B$ 76, $115427-5$ (2007).

2. D. B. Dougherty, P. Maksymovych, J. Lee, M. Feng, H. Petek, and J. T. Yates, Jr., "Tunneling spectroscopy of Stark-shifted image potential states on $\mathrm{Cu}$ and $\mathrm{Au}$ surfaces," Phys. Rev. B 76, 125428-9 (2007).

3. A. Winkelmann, V. Sametoglu, J. Zhao, A. Kubo, and H. Petek, "Angle-dependent study of a direct optical transition in the sp bands of $\mathrm{Ag}(111)$ by one- and two-photon photoemission," Phys. Rev. B 76, 195428-11 (2007).

4. M. Feng, J. Lee, J. Zhao, J. T. Yates, and H. Petek, "Nanoscale Templating of ClosePacked $\mathrm{C}_{60}$ Nanowires," J. Am. Chem. Soc. 129, 12394-5 (2007).

5. M. Feng, J. Zhao, and H. Petek, "Atomlike, Hollow-Core-Bound Molecular Orbitals of $\mathrm{C}_{60}$," Science 320, 359-62 (2008).

6. A. Winkelmann, W.-C. Lin, F. Bisio, H. Petek, and J. Kirschner, "Interferometric Control of Spin-Polarized Electron Populations at a Metal Surface Observed by Multiphoton Photoemission," Phys. Rev. Lett. 100, 206601-4 (2008).

7. J. Zhao, N. Pontius, A. Winkelmann, V. Sametoglu, A. Kubo, A. G. Borisov, D. Sanchez-Portal, V. M. Silkin, E. V. Chulkov, P. M. Echenique, and H. Petek, "Electronic potential of a chemisorption interface," Phys. Rev. B 78, 085419-7 (2008).

8. A. G. Borisov, V. Sametoglu, A. Winkelmann, A. Kubo, N. Pontius, J. Zhao, V. M. Silkin, J. P. Gauyacq, E. V. Chulkov, P. M. Echenique, and H. Petek, " $\pi$ Resonance of Chemisorbed Alkali Atoms on Noble Metals," Phys. Rev. Lett. 101, 266801-4 (2008).

9. S. Achilli, M. I. Trioni, V. Sametoglu, N. Pontius, A. Winkelmann, A. Kubo, J. Zhao, and $\mathrm{H}$. Petek, "Electronic structure of chemisorbed $\mathrm{Cs}$ and $\mathrm{Ba}$ atoms on $\mathrm{Cu}(111)$ from experiment and theory," Phys. Rev. B 80, xxx (2009). 
10. T. Huang, J. Zhao, M. Feng, H. Petek, S. Yang, and L. Dunsch, Phys. Rev. $B$ (submitted). 